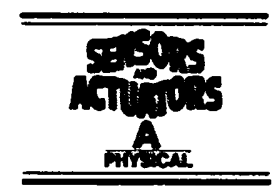

\title{
The future of biosensors
}

\author{
P. Bergueld \\ MESA Research Institute. University of Twente, Box 217, 7500 AE Enschede. Aietherlands
}

\begin{abstract}
Since the development of the glucose sensor by Clark and Lyons in 1962. generally recognized as the first biosensor, many types of sensors have been developed in which a physical or chemical transducer is provided with a layer containing a biological sensing element. The resulting device is called a biosensor, aimed to produce an electronic signal as a function of the concentration of a chemical or biochemical constituent of a liquid, not necessarily of biological origin. Among the many proposed concepts, the integration of biologically active mat:rials with a silicon chip is one of the most intriguing approaches, because it seems the most comprehensive integration between biology and electronics. In this paper the resulting biochips, mainly based on the field-effect principle as the coupling mechanism batween the two domains, will be described and discussed with an outlook on the future.
\end{abstract}

Keywords: Biosensors

\section{Introduction}

Biosensors consist principally of two basic components, connected in series: a molecular recognition system and a physicochemical transducer, as schematically given in Fig. 1 .

The division into two parts is not limited to biosensors, because any chemical sensor can in fact be divided into a selector part and a transducer part, although they are not always recognized as such. For example, potentiometric electrodes make use of selective interfacial chemical reactions between the electrode material and the liquid surroundings. leading to an interfacial potential. The transducer part is in this case very simple: a thermodynamically well-defined electrical contact to make the interfacial potential available for further handling. This action is usually called ditection and the transducer is therefore also referred to as a detector, most often connected to an electronic amplifier. In the case of biosensors the word receptor is often used for the actual recognition part of the sensor, because in the natural chemical senses, such as olfaction, taste and neural biochemical pathways, the recognition phenomenon is performed by chemoreceptor cells.

From a measurement point of view the interface between the receptor/selector and the transducer/detector part is very critical, because of the inherent sensitivity for interference from electrical fields in the case where the interface has a high electrical impedance. This becomes an even larger problem if relatively long leads are being used to connect the interface to a remote amplifier $c:$ impedance converter. This

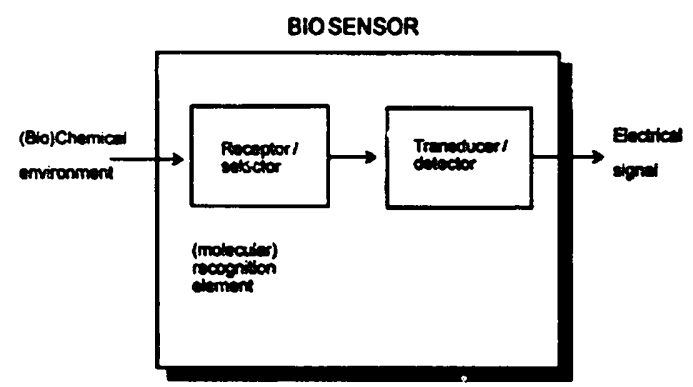

Fig. 1. Schemntic diggram of a biosensor.

measurement problem is one of the main reasons why in the field of chemical-sensor and biosensor research much attention is paid to the integration of the receptor function and the electronic function of the device. Since electronic functions are usually realized in silicon, the consequence is that silicon technology, or more cummonly photolitographic technology, was introduced in biosensor zesearch. In addition to the purpose of integration of electronic circuitry, the application of photolithography also appecured to be useful for shaping of electrodes and deposition and shaping of membranes with immobilized biological niolecules. Moreover, using the possibilities of silicon etching techniques, biosensor systems could be developed containing etched channels and cavities. This means that the original development of single biosensors, for instance, suitable for in vivo monitoring, gradually inuves towards the development of complete measurement 
systems, incorporated in one and the same chip. This development will be outlined in this paper in order to get insight into the different possibilities and pitfalls and thus into future expectations concerning the development of biochips.

Many examples can be found in the literature in which basic electronic components, such as FETs, thermistors, crystals, etc., with added biomolecular layers are used to create a specific biosensor. Because of the impact of ISFET development on biosensor chip development and because of the author's experience in this field, the above-mentioned future prospects will mainly be illustrated with examples of ISFET applications. Ultimately the ISFET forms one of the most elegant interfaces between an electronic device and a biological environment.

\section{The field effect as a sound base for biosensor chips}

The most critical point in information transfer from the (bio)chemical domain to the electrical domain is the actual interface between the two domains. A direct contaci between an ionic conductor and an electronic conductor is often not thermodynamically defined, resulting in an unstable interface potential and impedance, disturbing any desired measurement. On the other hand, many insulating metal and semiconductor oxides exhibit a stable contact with electrolytes. One of these oxides, e.g., silicon dioxide, when thermally grown on monocrystalline silicon, provides a stable contact with the silicon and thus with the electronic domain. The stabilization of the silicon dioxide-silicon interface is well known and fully exploited in MOS technology. Information transfer takes place by modulation of the electric field inside the oxide, resulting in a modulation of the so-called inversion layer in the silicon. This effect can be measured as a change in the resistance of a channel between a diffused source and drain region, which is the basic operation of the metal-oxidesemiconductor field-effect transistor (MOSFET), also referred to as insulated-gate field-effect transistor (IGFET), due to the insulating properties of the gate oxide. The technological development of the ISFET is fully compatible with MOS technology, with the exception that the electrical field inside the oxide is not controlled by an applied voltage to a metal gate electrode, but by a voltage applied to a reference electrode placed in the electrolyte in contact with the gate oxide. In addition, the electric field and thus the drain current is a function of the interfacial potential developed at the oxide/liquid junction, which is at least a function of the electrolyte $\mathrm{pH}$. This is in fact the selector part of the sensor.

From early ISFET experiments it is known that silicon dioxide is not the best $\mathrm{pH}$-selective material, nor does it provide a stable contact between the sensor and the liquid, resulting in an unacceptable drift behaviour. Therefore other oxides have been chosen for the contact with the electrolyte, resulting ultimately in ISFETs with an oxide sandwich. The inner oxide provides for a stable contact with the electrical domain. whereas the outer oxide provides for a stable contact

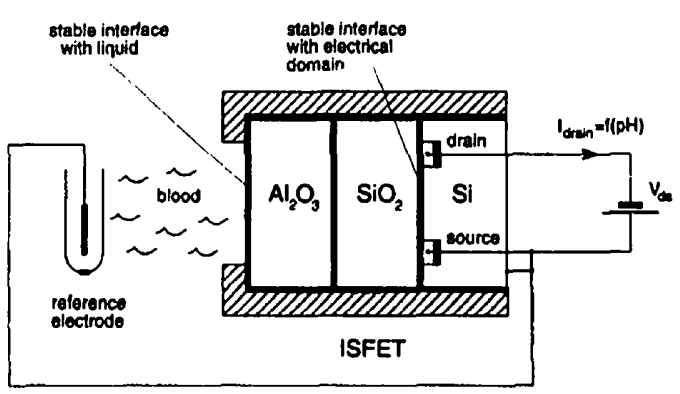

Fig. 2. Schematic representation of ISFET biosensor, focused on the interface requirements.

with the liquid. In addition, the outer surface has to be biocompatible in case the sensor is to be used for in vivo monitoring. The ultimate ISFET biosensor is schematically represented in Fig. 2.

Improvements of ISFET behaviour have mainly been focused on the improvement of the stability. Based on a theoretical description of the oxide interfacial physicochemical phenomena, specific oxides as well as a technology of oxide preparation, by which the optimal values of the theoretical key parameters were achieved, could be chosen.

The model that is nowadays generally accepted for the description of the oxide-solution interface, and with that of the $\mathrm{pH}$ sensitivity of an ISFET, is based on the site-binding model [1], originally developed and used in colloid chemistry [2].

The threshold voltage of an ISFET depends on the potential difference $\psi_{0}$ over the oxide-solution interface, which is the result of a charged oxide surface. The charging of the oxide surface can be explained by the site-binding model that describes the equilibrium between amphoteric $\mathrm{AOH}$ surface sites and $\mathrm{H}^{+}$ions in the solution, according to the following reactions: $\mathrm{AOH} \Leftrightarrow \mathrm{AO}^{-}+\mathrm{H}_{s}^{+}$and $\mathrm{AOH}_{2}^{+} \Leftrightarrow \mathrm{AOH}+\mathrm{H}_{\mathrm{s}}^{+}$, where $\mathrm{H}_{s}^{+}$refers to the protons in the direct vicinity of the surface. The resulting surface charge density is

$\sigma_{0}=q\left(n_{\mathrm{AOH}_{2}^{+}}-n_{\text {AO- }}\right)\left[\mathrm{C} \mathrm{m}^{-2}\right.$ j

with $q$ the unit charge and $n_{i}$ the number of surface $i$-sites per $\mathrm{m}^{2}$. As a result of this charge, an equal amount of charge $\sigma_{\mathrm{dl}}$, but with opposite sign, exists in the solution. These two charges form a capacitance, the integral double-layer capacitance $C_{\mathrm{dl}, i}$. The relation between $\sigma_{0}, \sigma_{\mathrm{dl}}, C_{\mathrm{dl}, 1}$ and $\psi_{0}$ is given by $\sigma_{0}=-\sigma_{\mathrm{d} 1}=C_{\mathrm{dl}, i} \psi_{0}$.

A general expression for the $\mathrm{pH}$ sensitivity of an ISFET can be derived, defined as the change in the potential $\psi_{0}$ over the oxide-solution interface as a response to a change in the bulk $\mathrm{pH}: \partial \psi_{0} / \partial \mathrm{pH}_{\mathrm{b}}\left[\mathrm{V} \mathrm{pH}^{-1}\right]$. This sensitivity is expressed in terms of the chemical intrinsic buffer capacity of the oxide surface and the electric capacitance of the double layer [3]. It can be derived that the differential intrinsic buffer capacity. $\boldsymbol{\beta}_{\text {inıd }}\left[\mathrm{m}^{-2}\right]$, equals

$\beta_{\mathrm{int,d}}=-\frac{1}{q}\left(\frac{\partial \sigma_{0}}{\partial \mathrm{pH}_{\mathrm{s}}}\right)$ 
where $\mathrm{pH}_{\mathrm{s}}$ refers to the $\mathrm{pH}$ at the oxide surface and the differential double-layer capacitance, $C_{\mathrm{dl}, \mathrm{d}}\left[\mathrm{F} \mathrm{m}^{-2}\right]$, can be expressed as

$C_{\mathrm{dl}, \mathrm{d}}=\frac{\partial \sigma_{0}}{\partial \psi_{0}}\left(=-\frac{\partial \sigma_{\mathrm{dl}}}{\partial \psi_{0}}\right)$

The combination of Eqs. (1) and (2) yields a simple expression for $\partial \psi_{0} / \partial \mathrm{pH}_{3}$, which can be related to the bulk by using the Boltzmann equation, resulting in the general expression for the $\mathrm{pH}$ sensitivity of an ISFET:

$\frac{\partial \psi_{0}}{\partial \mathrm{pH}_{\mathrm{b}}}=-2.3 \frac{R T}{F} \cdot \alpha$

with

$\alpha=\left(\frac{2.3 R T C_{\mathrm{dl}, \mathrm{d}}}{q^{2} \beta_{\mathrm{int,d}}}+1\right)^{-1}$

The parameter $\alpha$ is a dimensionless sensitivity parameter, varying between 0 and 1 , depending on the value of $\beta_{\text {int,d }}$ and $C_{\mathrm{dl}, \mathrm{d} \cdot}$

For oxide surfaces with a very high intrinsic buffe capacity the sensitivity parameter $\alpha$ approaches 1, resulting in the maximum sensitivity of $-59.2 \mathrm{mV} \mathrm{pH}^{-1}$ at $298 \mathrm{~K}$, the socalled Nernstian response. Under this condition the value of the double-layer capacitance and thus, according to the Debye-Hückel theory, of the electrolyte concentration is of no influence any more, resulting in an optimal selectivity for $\mathrm{pH}$. At the same time, this condition favours a surface that exhibits a very low interfacial impedance: the interfacial potential in fact behaves as an electromotive force.

The research concerning oxide/electrolyte interfacial behaviour should thus focus on maximal buffer capacity of the oxide, which can be reached with a maximum number of amphoteric surface sites with $\mathrm{p} K$ values of the acidic surface reactions close together. On the other hand, the technology to make these oxides should be compatible with MOS technology. Through the years different oxides as well as technologies to make these oxides have been tested. In practice tantalum pentoxide behaves nearly ideally and combines $\mathrm{pH}$ selectivity with a maximal sensitivity and a minimal drift. This is illustrated in Fig. 3.

From the results shown in Fig. 3 it may be concluded that at present ISFET behaviour can compete in all aspects with that of conventional $\mathrm{pH}$ glass membrane electrodes, winich might be the reason why ISFET $\mathrm{pH}$ probes are now offered for sale by many companies in Europe. Japan and the USA. As a result of this improved behaviuur, the ISFET is veell suited for use as a stable transducer for biosensors which are based on a recognition system that induces a local $\mathrm{pH}$ change in a membrane deposited on the ISFET surface and containing the receptor molecules. In this respect advantage can be taken of natural biochemical reactions which often induce a change in proton concentration, as in many enzymatic and immunoreactions. The fast response of an ISFET to local pH changes is another advantage of the combination.
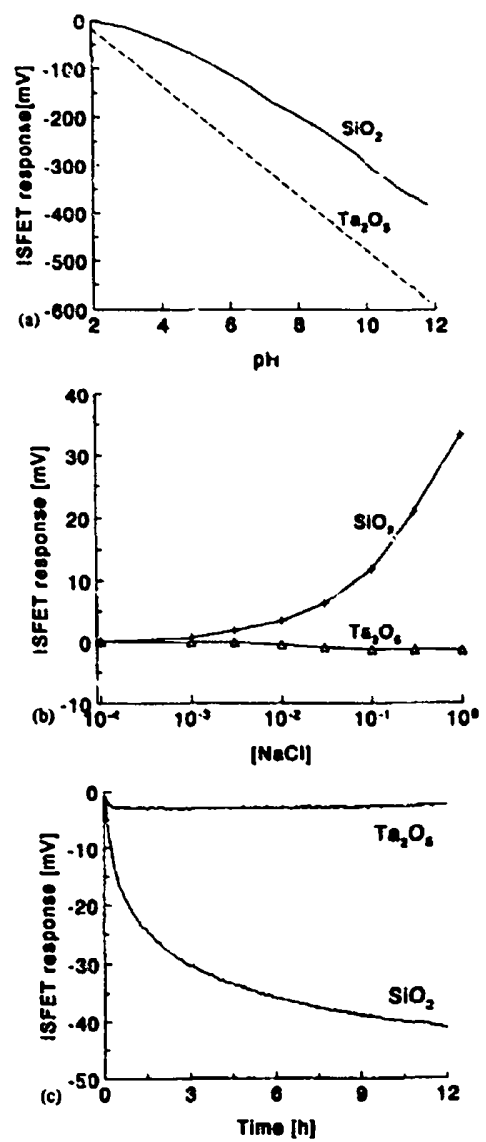

Fig. 3. (a) pH sensitivity of silicon dioxide and tantalum pentoxide at 0.1 $\mathrm{M}$ TBACl. (b) Influence of $\mathrm{NaCl}$ on both oxides at $\mathrm{pH}=5.8$. (c) Drift behaviour of both oxides.

\section{The measurement concept}

The conventional approach to measuring a non-electrical parameter with a sensor is based on one-directional information transfer from the physical or chemical domain towards the electrical domain. This method is schematically shown in Fig. 4(a). In Section 2 it was shown how improvements in the applied materials and technology can lead to improvements in the stability of the information transfer. Regardless of the necessity to continue the efforts to improve a sensor's stability, it should be mentioned that sensor properties will never be constant for ever. This means that, depending on experience in relation to the required accuracy and reliability for a specific measurement, the sensor should be (re)calibrated regularly.

The question arises whether sensors using silicon technology are favourable in this respect. For ISFETs the possibilities are sketched in Fig. $4(\mathrm{~b})$. It shows that now information can aiso be sent to the sensor, for instance, to reset its baseline to 


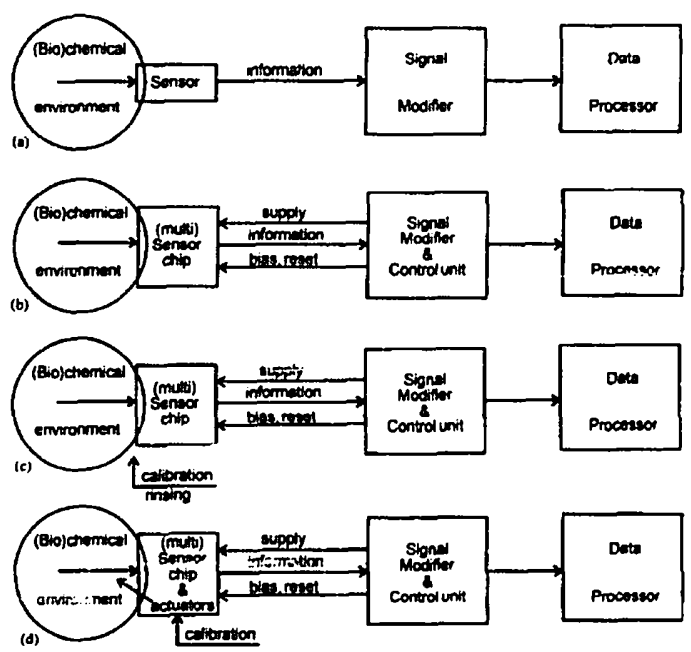

Fig. 4. Schematic representations of: (a) conventional sensor measurement set-up; (b) silicon chip multisensor measurement set-up: (c) biosensor measurement set-up; (d) a sensor with integrated solid-state actuator.

a predetermined level. This is only worthwhile if this action can be deduced from the sensor's behaviour during a measurement. Often additional information is necessary for the control unit, for instance, the temperature of the sensor. In this case the application of silicon technology is favourable over other technologies, because in silicon many sensors can easily be integrated. In general it can be expected that multi sensing of parameters delivers more information, which can be used in combination with an appropriate algorithm by the control unit to keep the sensor characteristics automatically within an acceptable range. This is of course a well-known and generally applied concept in making electronic functions operate within specifications independent of time and temperature. However, for chip-based chemical and biosensor systems this bi-directional information transfer is not enough, because it does not imply the proper functioning of the recognition system. The activity of biomolecules will certainly change with time and be a function of the (changing) composition of the surrounding liquid. Therefore, the procedure of regular recalibration is still necessary, as schematically shown in Fig. 4(c). Not only a calibration liquid has to be used, but often also a rinsing liquid to prevent possible fouling of the biosensor interface.

It will be clear that for accurate and reliable measurements with a biosensor, the set-up as given in Fig. 4(c) will be necessary. However, this leads to more complex systems than originally expected when the first developments of biosensors started. It will also be clear that implantation of biosensors in human beings is not a realistic possibility when using the approach sketched in Fig. 4(c). Accordingly, the systems as described in the literature over the last decade are not aimed at this application, but intended for temporary diagnostic measurements, for instance, during an operation. Since measurement and rinsing/calibration cycles are executed succes- sively, this type of measurement is in principle based on a sampling technique. The most promising developments are given below.

Based on the original research of Sibbald at the University of Newcastle upon Tyne, the Central Research Laboratories of Thorn EMI developed a microprocessor-controlled ISFET analysis instrument [4]. Besides the control unit the instrument contains pump motors. A video-cassette-sized unit can be attached to the instrument, containing pump spindles, ISFET sensors, calibrant and flushing solutions as well as a waste container. The quantities of snlution required for each measurement are very small and all liquids are passed to the waste container. The cassette unit holds enough calibrant solutions for about $\mathbf{2 0 0}$ measurements. After this the cassette has to be replaced by a fresh one. The instrument is tested as a bedside clinical blood analyser for $\mathrm{pH}, \mathrm{pNa}, \mathrm{pK}$ and $\mathrm{pCa}$ and can handle unheparinized blood samples.

Although not developed as far as the aforementioned cassette, the University of Neuchatel has developed a flowthrough cell for the measurement of $\mathrm{pH}, p \mathrm{CO}_{2}$ and $p \mathrm{O}_{2}$ [5]. The first parameters are measured with an ISFET-based sensor and the last one with an amperometric sensor. The liquid channel containing the sensors is $18 \mathrm{~mm}$ in length and $2 \mathrm{~mm}$ in width, resulting in an internal volume of only $5 \mu$. In total nine sensors are integrated to receive redundancy in the measurement in order to increase the reliability of the system. This system, shown in Fig. 5, can serve as the heart of a micro total analysis system ( $\mu$ TAS).

At Siemens AG in Erlangen, Gumbrecht et al. developed a system based on a double-lumen catheter [6], the outer lumen being longer than the inner one, connected to two pumps as schematically represented in Fig. 6.

Inserted into a blood vessel, the outer lumen is first flushed with an air-saturated and $\mathrm{pH}$-buffered calibration solution by pump nr. 2. Due to the higher flow rate of pump nr. 2 compared to that of pump nr. 1 and the shorter inner lumen, the calibration solution is drawn through the inner lumen along the sensor array in the external flow-through cell. This cell contains, for instance, two pH-sensitive ISFETs at a certain

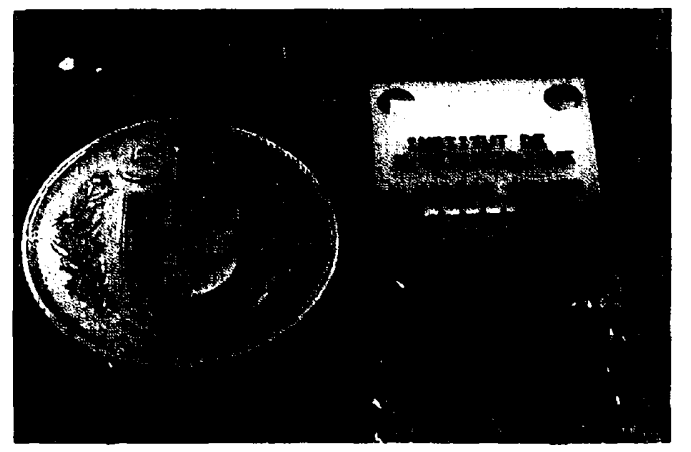

Fig. S. Photograph of the sensor system developed at the University of Neuchatel, together with a Swiss 5 Franc coin. The arrow indicates the liquid channel. 

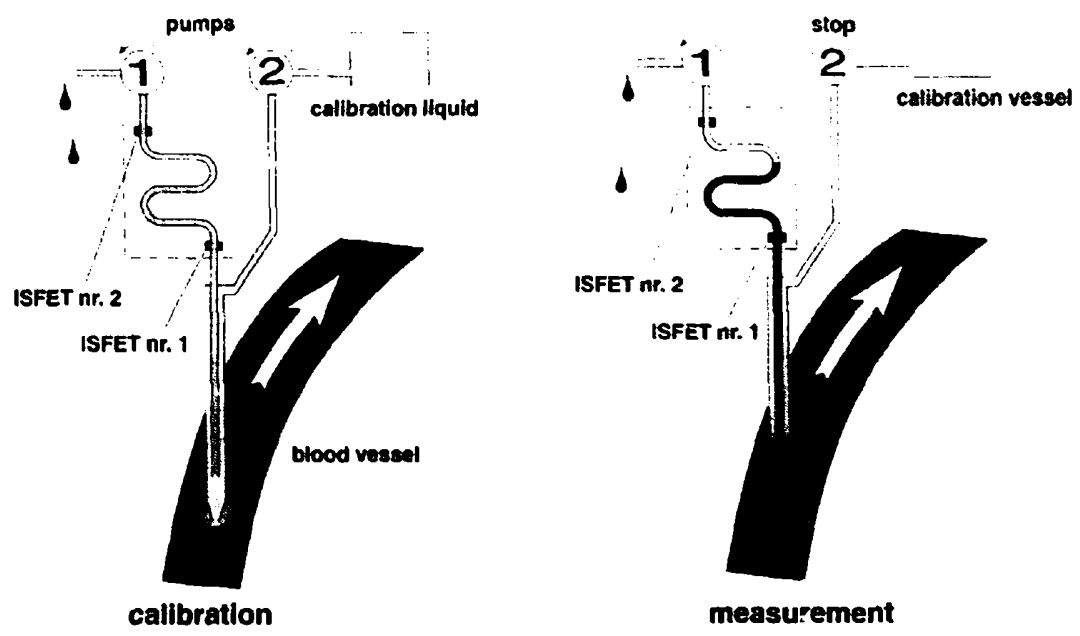

Fig. 6. Calibration and ineasurement cycle of the Gumbrecht blood sampling and analysis system.

distance. In this calibration cycle both ISFETs are in contact with one and the same calibration solution and should thus record the same $\mathrm{pH}$ value. If not, the control unit can reset the sensors. Interruption of pump nr. 2 allows blood to enter the cell, initially contacting the first ISFET, later the second one. This means that in two successive time intervals temporarily the blood $\mathrm{pH}$ is measured with respect to that of the calibration solution. The cycle can be repeated continuously, for instance once every two minutes, and the controlled pump speeds dictate the timing and interpretation of the recordings. The system uses only $10 \mathrm{ml}$ blood and $5 \mathrm{ml}$ calibration solution per $24 \mathrm{~h}$ and has also been built with a three-electrode amperometric $p \mathrm{O}_{2}$ sensor added to the ISFETs.

The previous examples show how in practice the system approach, as schematically shown in Fig. 4 (c), has been adapted to create a system for continuous measurements as originally thought to be the ultimate possibility of biosensor chips. An alternative to the on-line measurement approacb is the one-shot measurement, as known from the glucose , $n$. For the simultaneous measurement of glucose, urea, sodium,

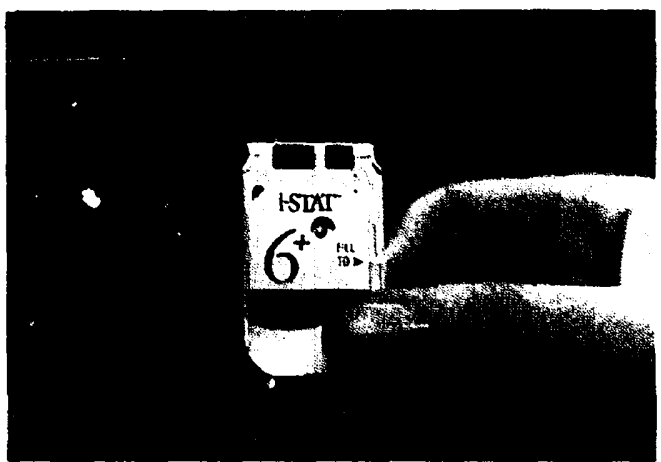

Fig. 7. Photograph of the disposable biosensor carridge developed by the company i-STAT. potassium, chloride, hematocrit and haemoglobin, the company i-STAT in Princeton, USA, developed a disposable cartridg with a size of $45 \mathrm{~mm} \times 27 \mathrm{~mm} \times 5 \mathrm{~mm}$, containing the relevant biosensors, a calibration solution and a waste container [7], as shown in Fig. 7. A blood sample has to be inserted in the sample inlet by hand. Then the cartridge is inserted into a portable clinical analyser for processing. The analyser makes electrical contact with the cartridge and automatically recognizes the test to be run. Before the actual measurement, the calibrant solution is sent over the biosensor array. After calibration the blood sample is automatically sent over the sensors and the analyser displays and stores the measured data.

In all measurement concepts described above use is made of a flow cell with integrated biosensors, whilst the system operates with controlled transport of liquids. This means that liquid containers and a transport mechanism are essentiai parts of the system. This not only makes the systems rather complex but it also limits the operational time. It would therefore be useful to develop a system which does not need these additional calibration liquids, but nevertheless has the same possibilities of intermediate testing. With this desire in mind, the author of this paper started to develop solid-state actuators, to be integrated with the sensors and to be applied on an in situ base. This concept is schematically illustrated in Fig. 4(d).

With respect to a pH-sensitive ISFET sensor, the matching pH actuator can be developed relatively easily, because protons can be generated coulometrically at a noble metal electrode which is shaped around and/or above the gate of an ISFET. When a current (pulse) is applied to the actuator electrode with respect to a remote counter electrode, the hydrolysis of water will result in a $\Delta \mathrm{pH}$ in the direct vicinity of the gate. In this way a coulometrically induced very local utration can be performed in situ within some seconds, with current values of the order of $10 \mu \mathrm{A}$. In a closed system (the 
actuator is situated above the gate, for instance, as a porous electrode) the end point of the titration is linearly related to the acid or base concentration of the analyte, whereas in an open system it is a square-root relation [8]. Note that in this type of concentration measurement no longer is the absolute value of the ISFET amplifier output voltage the measurand, but the time lag between the onset of the current pulse and the end point in the titration. This means that possible drift in the baseline and sensitivity of the ISFET is no longer of importance and the system can thus be used continuously without any recalibration for much longer periods of time than in the case of static measurements. In that respect the system is suitable for implantable use, because it does not need any calibration liquids. However, since chloride ions may interfere with the hydrolysis of water, for in vivo use the noble metal electrode has to be replaced by an electroactive material, such as irOx [9]. Althuugh an acid-ibase actuator was easy to find, it may become a problem to develop appropriate actuators for other species. Therefore, more attention should be paid to the development of different types of chemical actuators, for instance, based on electrically controlled release of species from certain polymers as in use in pharmacology.

Summarizing this section on measurement concepts, it should be noted that the application of biosensurs moves from single use towards a system approach, in which case the necessary introduction of liquid-handling systems may be circumvented by the application of solid-state actuators. This will, however, need a fair future research effort.

\section{Dynamic measurements for biomolecular recognition}

In Section 3 the conventional approach of a static measurement has been abandoned and replaced by a dynamic measurement in order to circumvent the problem of calibration and recalibration. The cxamples were limited to the measurement of rather simple electrolyte components. The most intriguing development in the field of biosensors is, however, the measurement of a large quantity of different biomolecules. The most selective recognition system in nature is that of the immune reactions, which resulted in many attempts to develop biosensors in which the actual recognition takes place in a layer of antibodies at the surface of the sensor. In general these sensors with a biomolecular recognition system are referred to as bioaffinity sensors. Since in principle FET devices measure a surface charge and since antibodies, or more generally, protein molecules are mostly charged molecules, it has long been expected that the simple adsorption of a layer of antibody molecules at the surface of an ISFET follower by the selective binding to it of antigen molecules would be a unique possibility to sense biomolecules. This imaginary device was calied an ImmunoFET (IMFET), but its operation has never been proven to exist. The reason is of course that no net charge is available, because the surface charges of protein molecules are neutraiized by ever-present counter ions. The resulting double layer, with a thickness of the Debye length, is of the order of $1 \mathrm{~nm}$ thick in moderate electrolyte concentrations. Beyond this distance no external electric field exists. Hence the idea that a layer of charged molecules at the surface of an ISFET modulates the electric field in the gate oxide should definitely be forgotten [10].

Another idea was that protein molecules adsorbed in a porous membrane deposited on an ISFET surface would modulate the gate input potential due to the existence of a Donnan potential. Although this idea also appeared not to be directly applicable as far as static measurements are concerned, the idea of exploiting the Donnan phenomenon in a dynamic way is quite interesting. To understand the Donna.i phenomenon, which was first recognized by Donnan in 1911, the classical theory has to be summarized. In this theory the potential between two aqueous solutions separated by a semi-permeable membrane is calculated. Both solutions contain a dissociable salt, for instance $\mathrm{NaCl}$, of which in one solution the $\mathrm{Na}^{+}$ions are partly replaced by an equivalent amount of positive charges on protein molecules. The membrane is supposed $t o$ be permeable for the small cations and anions, but not for the protein molecules. The ssdium ions will diffuse through the membrane from the solution without protein molecules due to the concentration gradient, initially tracking the chloride ions even against the developing concentration gradient. Donnan proved that the diffusion of both types of ions is in equilibrium as soon as the concentration ratios for $\mathrm{Na}^{+}$ and $\mathrm{Cl}^{-}$ions, $c_{\mathrm{Na}^{+}}$and $c_{\mathrm{Cl}^{-}}$, have become reciprccal in the two solutions:

$\frac{c_{\mathrm{Na}}+(1)}{c_{\mathrm{Na}}+(2)}=\frac{c_{\mathrm{Cl}-}(2)}{c_{\mathrm{Cl}}-(1)}=r_{\mathrm{D}}$

where (1) and ( 2 ; refer to the two solutions and $r_{\mathrm{D}}$ is the socalled Donnan equilibrium constant (the activity coefficient is assumed to be unity). Besides a difference in osmotic pressure between the two solutions, an electrostatic potential differe:lce is established over the membrane to prevent a net transport of ions. Its value is given by the Nernst formula:

$\phi_{\mathrm{D}}=\frac{R T}{F} \ln \frac{c_{\mathrm{Na}^{+}}(1)}{c_{\mathrm{Na}^{+}}(2)}=\frac{R T}{F} \ln r_{\mathrm{D}}$

From this theory it can be concluded that the Donnan phenomenon results in an electrical potential over a junction between two solutions, one of which contains immobilized protein molecules, as may be the case in a membrane, for instance. In addition the theory predicts that as well as this electrical potential a redistribution of all diffusible ions takes place, including the protons. With respect to a device consisting of a protein-containing membrane deposited onto a pH-sensitive ISFET, two possibiiities are now manifest. If the membrane/ISFET interface consists of a very thin electrolyte interlayer, which is often the case in practice, the membrane develops a Donnan potential of the same magnitude at both the external and the internal interface. The result 
is that the ISFET does not measure any effect, because both potentials cancel each other. If the membrane is very closely adsorbed or covalently bound to the ISFET surface and no electrolytic interlayer exists, the ISFET measures the external Donnan potential, but also the membrane pH. Eqs. (5) and (6) applied to the proton distribution predict that the membrane $\mathrm{pH}$ is adapted with respect to the bulk $\mathrm{pH}$ in a Nernstian way. Eq. (3) predicts that for an ISFET with $\alpha=1$ the ISFET will respond in a Nernstian way to this adapted membrane $\mathrm{pH}$. The result is that the overall response is zero, because the adapted membrane $\mathrm{pH}$ just opposes the Donnan potential. Only in the case that an ISFET with $\alpha \neq 1$ is used will a net response bc measured, but unfortunately thisi iy pes of ISFETs are less stable and thus not suitable for static measurements.

From the previous considerations it should be concluded that static measurements of Donnan-induced effects can not or hardly be made. An alternative approach is, however, to exploit the Donnan effect of protein molecties on a molecular level instead of on the macro level as in the membrane phase described above. Considering a protein molecule as an impenetrable sphere, occupied by discrete ionizable groups such as amino and carboxyl groups, the theory as given for the operational mechanism of ISFET surfaces (Eqs. (1)(3) ) can be applied. Also a protein surface as defined in the sphere model exhibits a surface potential and a related suríace chars,e, coupled by the double-layer capacitance:

$\sigma_{\mathrm{s}}=\psi_{\mathrm{s}} C_{\mathrm{d} \mathrm{t}}$

For ISFET surfaces as well as protein 'surfaces' Eq. (7) prescribes that an externally induced change in the value of the clouble-layer capacitance will result in an adaptation of the surface charge, because the surface potential remains determined by the bulk $\mathrm{pH}$. The doubie-layer capacitance can be changed by changing the electrolyte concentration as described by the Debye-Hückel theory. The larger the concentration, the smaller the Debye length and thus the larger the double-layer capacitance. With a sudden step in the electrolyte concentration, the double-layer capacitance changes nearly instantly, but the charge adaptation will be retarded due to the acid-base reaction kinetics of the surface groups. In order to obey Eq. (7), the surface potential will therefore temporarily be out of equilibrium and can only recover to its equilibrium values as determined by the bulk $\mathrm{pH}$ by letting the surface groups take up or release protons. Both ISFETs and protein molecules react in this way to a sudden change in electrolyte concentration. Because at $\mathrm{pH}=7$ tantalum pentoxide is negatively charged, the response to an ion step is a positive potential transient (Fig. 8, curve 1). Modifying the surface with a monolayer of a protein, for instance protamine, the added groups, with in this case a net positive charge, lead to a response on the same stimulus which is less positive (Fig. 8, curve 2). After incubation of the device with heparin, which strongly binds to the protamine and modifies the surface again by the introduction of negative groups, the response is again more positive (Fig. 8, curve 3 ).

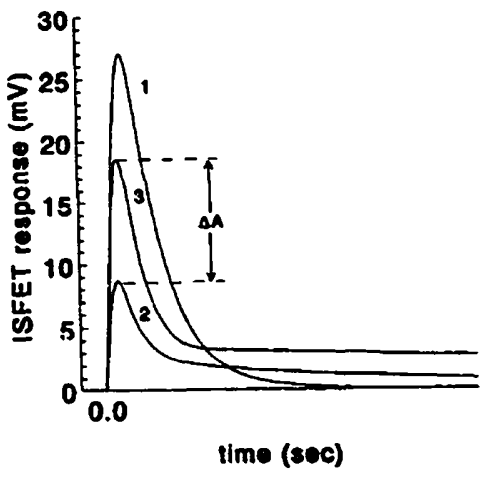

Fig. 8. Typical responses to an ion step of 10 to $100 \mathrm{mM} \mathrm{KCl}$ at pH 7. Cerve 1 represents the response of a bare ISFET, curve 2 of the same ISFET with a monolayer of protanine.

By subsequent incubations of only $2 \mathrm{~min}$ in solutions having different heparin concentrations, with regeneration steps in $5 \mathrm{M} \mathrm{NaCl}$ after each measurement, a linear relation could be found between $\boldsymbol{\Delta A}$ (amplitude difference between transient 2 and 3) and the heparin concentration in $\mathrm{U} \mathrm{ml}^{-1}$ [11]. Similar measurements were done with other systems, where the protamine molecules were adsorbed in a porous membrane consisting of polystyrene beads in an agarose gel [12]. Also immunoreactions could be monitored in this way [13].

Note that the stimi Jus/response experiment with the protein-covered ISFETs as well as the coulometric titration as described in Sertion 3 explicitly make use of a dynamic measurement concept as the ultimate solution for ine problems inherent to static measurements.

\section{Future biosensor system concepts}

Combining the system approach as schematically shown in Fig. 4(c) and (d) and illustrated with examples in Section 3 with the dynamic stimulus/response approach as described above may in the future lead to a new type of biosensor system. However, the inplementation of an ion step may complicate the practical performance, because in addition to the biosensor it needs a rather complicated liquidhandling system. It may however be expected that liquidhandling systems can in the future be integrated in a silicon chip or at least in the system substrate, a development presently under investigation as the $\mu$ TAS (micro total analysis system) [14]. An alternative may be the introduction of another type of stimulus, preferably in the physical domain, to disturb an equilibrium temporarily. A temperature step or a current through an actuator may be possible. The latter approach has already been shown to be possible and is called chronopotentiometry [15].

Biocompatibility problems may be circumvented by combination of the sensor system with the microdialysis tech- 
nique. Fundamental research in this direction has, however, been siarted only recen: $y$.

\section{Future realization oi recognition systems}

A problem with all biosensor systems may be the limited lifetime of th: : iomolecules used as receptor. As the technology used to produce the transducer part of the sensor is solid in the sense that it will in principle remain stable for ever, this is not the case with biological materials. So the question may arise whether it is realistic to expect that biosensors can be produced in a factory and after a certain shelf life be brought into contact with a biologically or chemnically active surrounding and then operate continuously for a long. term measurement. If not, it may be expected that the biosensor systems can only be used for a one-shot measurement (disposable use). An escape from this rather disappointing conclusion may result from the considerations generated by the following experiment.

In order to analyse the cause of accelerated caries with xerostomia patients, ISFETs were mounted in a dental prosthesis and first tested in volunteers [16]. During the first days of wearing the prosthesis the ISFET recording was rather noisy. However, the measurement stabilized during the fourth day. Rinsing the mouth with a little glucose water showed a dramatic decrease of the pll, from 7 to 4 . By chewing, the $\mathrm{pH}$ could again be restored to the initial value. This was not the case with xerostomia patients. There mouth $\mathrm{pH}$ remained at the low value of 4 , which is thought to be the cause of the accelerated caries. Analysing the measurement in more detail, it was concluded that initially the noisy signal was caused by the local variation in the saliva $\mathrm{pH}$. At the fourth day it is assumed that a dental plaque has been formed on the teeth and on the ISFET surface. The bacteria in this plaque provide tor a certain buffer capacity in the micro surroundings of the ISFET, thus preventing the saliva $\mathrm{pH}$ changes from being measured. A stable recording is the result. Upon feeding the bacteria with glucose, they produce acids and the corresponding $\mathrm{pH}$ chan ?, is directly measured by the adjacent ISFET. In fact the ISFET in the mouth is transformed into a glucose sensor. Unfortunately the hacteria are not selective for glucose, so this approach does not directly lead to a new type of glucose sensor. Nevertheless, the way of constructing the biosensor is interesting: a solid-state transducer was constructed with human technology and as such implanted in the human body, which reacted by depositing a bacterial bioreactor on its surface. The finishing touch, so to say, was given by the in vivo surroundiligs, after which the transducer was transformed inio a biosensor. This line of reasoning may lead to a whole new approach to manufacturing biosensors. Only a semi-manufactured product has to be produced and implanted in the biological surroundings in which a measurement should take place and nature will do the rest. An advantage of this approach is that the biologically active material used for the recognition is by nature biocompatible and keeps itself alive. This development may ultimately lead to the existence of 'real' biosensors.

\section{Conclusions}

Along the lines of the earlier and present developments in the field of biosensor research and applications, it has been shown that the system approach gains in importance. Measurements with a single biosensor struggle with stability and calibration problems, mainly caused by the fact that a static measurenient is most often performed. With the system approach dynamic measurements are possible, which dc not have these problems. When suitable solid-state actuators for chemical species can be developed, dynamic measurements will gain in significance. With respect to the lifetime and stability of the recognition system, making the function in situ is an interesting philosophy.

\section{Acknowledgements}

The author acknowledges the Dutch Tech: ology Founjation (STW) and the Dutch Foundation for Research on Matter (FOM) for their support in different projects in the field of biosensor research, by which especially dynamic measurement concepts could be developed.

\section{References}

[1] L.J. Bousse, N.F. de Rooij and P. Bergueld, Operation of chemically sensitive field-effect sensors as a function of the insulator-electrolyte interface, IEEE Trans. Electron Devices, ED-30 10 (1983) 12631270.

[2] D.E. Yates, S. Levine and T.W. Healy, Site-binding model of the electrical double layer at the oxide/water interface, $I$. Chem. Soc. Faraday Trans., 70 (1974) 1807-1818.

[3] R.E.G. van Hal, J.C.T. Eijkel und P. Bergveld, A novel description of ISFET sensitivity with the buffer capacity and double-fayer capacitance as key parameters, Sensors and Actuators B, 24-25 (1995) 201-2C5.

[4] I. Robins, Sensing developments using ISFETs, Intermational LABMATE ( 1993) 45-46.

[5] P. Arquint, M. Koudelka-Hep, B.H. van der Shoot, P. van der Wal and N.F. de Rooij. Micromachined analyzers on a silicon chip, Clin. Chem., 40 (1994) 1805-1809.

16] W. Gumbrecht, W. Schelter and B. Montag, On line blood electrolyte monitoring with a ChemFET microcell system. Sensors and Actuators. BI (1990) 477-488.

[7] E. Jacohs, E. Vadasdi, L. Sarkozi and N. Colman. Analytical evaluation of $\mathrm{i}$-STAT portable clinical analyzer and use by non-laboratory healthcare professionals, Clin. Chem., 39 (1993) 1069-1074.

[8] W. Olthuis and P. Bergveld, Integrated coulometric sensor-actuator devices, Microchimica Acta. 121 (1995) 191-223.

[9] W. Olthuis, J.G. Bomer, P. Bergveld, M. Bos and W.E. van der Linden, Iridium oxide as actuator material for the ISFET-based sensor-actuator system. Sensurs and Actuators B. 5 (1991) 47-52.

[10] P. Bergveld. A critical evaluation of direct electrical protein detection methods, Biosensors Bioelectron., 6 (1991) 55-72. 
[11] J.C. van Kerkhof, P. Bergveld and R.B.M. Schasfoor, The ISFETbased heparin sensor with a monolayer of protamine as affinit; ligand. Biosensors Bioelectron.. 10 (1995) 269-282.

[12] J.C. van Kerkhof, P. Bergveld and R.B.M. Schasfourt, Development of an ISFET-based heparin sensor using the ion-step measuring method, Biosensors Bioelectron., 8 (1993) 463-472.

[13] R.B.M. Schasfoort, C.E.J.M. Keldermans, R.P.H. Kooyman, P. Bergveld and J. Greve, Competitive immunological detection of progesterone by means of the ion-step induced response of an immunoFET, Sensors and Actuators, Bl (1990) 368-372.
[14] A. van der Berg and P. Bergveld (eds.). Micro Tosal Analysis Systems. Proc. MicroTAS W4 Workshop, Kluwer. Dordrecht. 1994.

[15] P. Bergveld, J.C.T. Eijkel and W. Olthuis, Detection of protein concentrations with chronopotentiometry. Biosensors Bioelectrum. submitted for publication.

[16] L.L. Visch, P. Bergveld. W. Lamprecht and E.J. 's-Gravenmade, pH measurements with an ion-sensitive field-effect transistor in the mouth of parients with xerostomia, IEEE Trans. Biomed. Eng.. 38 (1991) 353-357. 